\title{
MÁS ALLÁ DE LA CONCIENCIA DEL “TERCER MUNDO" EN UN FEMINISMO SIN FRONTERAS
}

\author{
BEYOND THE AWARENESS OF THE “THIRD WORLD” IN A FEMINISM \\ WITHOUT FRONTIERS
}

Xiana Sotelo

Universidad Complutense de Madrid

\section{Resumen:}

Ante una sociedad principalmente patriarcal, una serie de mujeres alzaron sus voces para proclamar igualdad y encontrar sus identidades, tras verse indefensas e incomprendidas en sus países, en medio de una sociedad patriarcal. Mujeres que con valentía hicieron que sus voces fueran oídas en zonas más discriminadas, lo que conocemos como feminismo "del tercer mundo".

\section{Palabras claves:}

Tercer mundo, feminismo, mujer.

\section{Abstract:}

Before a principally patriarch society, several women raised their voices in order to proclaim equality and find their identities, after having seen themselves as defenceless and misunderstood women in their own countries, in the middle of a patriarchal society. They were brave women whose voices were heard in the most discriminated areas, what it is known as feminism "of the third world".

\section{KEY WORD:}

Third world, feminism, woman. 
Trazando las huellas de una sociedad patriarcal, imperialista, capitalista y heterosexual, el revisionismo feminista está en deuda con el Feminismo del Tercer Mundo. (“Third World Feminism").

Desde la década de los 80s, feministas asio-americanas, afro-americanas, nativoamericanas chicanas y latino-americanas, han defendido la etiqueta social y académica de "Mujeres de Color" como reivindicación al racismo, clasismo e imposición heterosexual dentro del movimiento feminista anglo-europeo. Reconociendo sus lazos históricos con países colonizados despertaron a la conciencia del tercer mundo revolucionando así, el dominio académico del discurso de la identidad política y la resistencia cultural, reclamando la especificidad cultural como premisa básica en la apertura de un diálogo intercultural.

Era el amanecer de una nueva conciencia crítica post-feminista; el nacimiento de unos poderes sociales y culturales donde nunca antes los había habido. Decididas a hacer de la revolución del pensamiento crítico algo totalmente irresistible ${ }^{1}$, levantaron puentes con sus propias espaldas y utilizaron sus palabras como escudo y como antídoto.

El feminismo "del tercer mundo" a través de las diferentes ediciones (1981, 1983, 2001, 2002) de la primera antología de "mujeres de color" This Bridge Called My Back: Writings by Radical Women of Color, ha redefinido la búsqueda de lo político de la "identidad" y la "diferencia" en un encuentro intercultural no jerárquico gracias a que han destapado el imperialismo inherente en el himno occidental de la "mujer universal" y su canto a un "feminismo global". Así, cuando en 1920 Virginia Wolf en The Three Gineas declaraba que: "As a woman I have no country. As a woman my country is the whole world",sus palabras le coronaron como la portavoz de un anhelado desafío público a un mundo de culturas y tradiciones sexistas, pero a la vez, se olvidaron de matizar que la categoría política de "mujer" a la cual ella representaba, formaba parte de una élite colonizadora.

¿Por qué de repente se empezó a escuchar a las mujeres después de siglos de esta silenciadas por la mentalidad reduccionista de las religiones patriarcales?

No debemos olvidar que fue gracias a la coyuntura de las colonias europeas que las primeras mujeres anglo-europeas pudieron convertirse en "full individuals" a expensas de la complicidad cultural de pertenecer a un colectivo socialmente poderoso, que la feminista teórica Rosemary Marangoly George ha bautizado como “Raza Master"2.

1 Palabras de la escritora afro-americana Toni Cade Bambara "1981 Foreword"en This Bridge Called My Back: Writings by Radical Women of Color . Moraga, Cherrie y Gloria ALzandúa., eds. Watertown, M.A. Persephone: xLiii.

2 Ensayo Rosemary M. George, "Holmes in the Empire, Holmes in the Home"en Burning Down the House. Recycling Domesticity. Columbia Press, 1998. p.48
La feminidad desmembrada por el yugo patriarcal alcanzó un "Yo autoritario" (authoritative self) que solo pudo ser posible gracias a que su "identidad" empezó a definirse en contraposición a un "racial Other"3; es decir, ellas eran de una "raza master" en presencia de grupos colonizados, tanto mujeres como hombres ${ }^{4}$.

En ese primer despegue feminista, “las mujeres occidentales” que se revolvían contra la opresión de género, es decir, las primeras feministas públicamente reconocidas, tuvieron en sus manos la oportunidad de levantar alianzas étnicas y anti-clasistas en el nombre de la "universalidad de la opresión", que podría definirse como todas aquellas caras en las que la opresión se despliega y todos aquellos medios por lo cuales se ejecuta y es sentida tanto personal, como colectivamente. Pero no fue así El feminismo se traicionó a sí mismo, olvidándose de que no se puede esculpir en el imaginario colectivo a una "mujer universal" sin que todas las mujeres del mundo estén interculturalmente unidas.

Volver hacia atrás y contextualizar los orígenes de una identidad feminista moderna como el resultado de los entresijos de un juego de poderes, es calibrar la verdad histórica de que "the modern individual was first and foremost an imperialist"

Es por ello que podía decirse que dentro del diálogo intercultural la significación moderna de "inferioridad" se remonta a la dialéctica de una "raza master".

Y aunque en la quimera de una justicia social basada en la igualdad de género, el feminismo anglo-europeo lideró la rebelión de valores y sistemas de creencias artificialmente ideados y culturalmente impuestos para sostener patriarcados, la "mujer de la raza master", a través de su afiliación cultural a un grupo históricamente colonizador, en el marco de la cooperación y las relaciones interculturales, al no establecer alianzas étnicas y de clase no solo con el colectivo de mujeres "colonizadas" sino con todos los colectivos oprimidos, ha ayudado a mantener una dinámica neocolonizadora que ha hecho perder credibilidad al espíritu inclusivo de "lo feminista" y que en una progresiva radicalización del pensamiento ha reducido la opresión feminista a una cuestión de género

Es por ello que cuando en 1974, "The Black Feminist Statement" 6 del Combahee River Collective, escrito por un grupo de mujeres feministas de Boston inspirado en el movimiento por los derechos humanos y civiles que partió de los EEUU en los años 60s, cambiaría el curso de la praxis académica feminista, al emerger

\section{Ibid. \\ 4 Ibid.p.57}

5 Rosemary M. George, "Holmes in the Empire, Holmes in the Home" p.49

6 Escrito a petición de Zillah Einsten, para incluirlo en su antología Capitalist Patriarchy and the Case for Socialist Feminism y mayormente difundido gracias a su aparición dentro de la antología de "mujeres de color" This Bridge We Called Home: Writings by Radical Women of Color. 
socialmente como la "Contra-raza master" (Counter-Master Race) y destapar la falta de solidaridad dentro de un movimiento que supuestamente debería estar luchando en su nombre. Dejando claro que estaban denunciando públicamente el racismo dentro del movimiento feminista, con un énfasis en las intersecciones de raza, sexo y clase como factores simultáneos de la opresión declaraban que: "We are actively committed to struggling against racial, sexual, heterosexual and class oppression and see as our particular task the development of integrated analysis and practice based upon the fact that the major systems of oppression are interlocking "."

Evidenciando como los proceso de globalización económica y el capital transnacional rigen el pulso económico y político de un mundo polarizado en la división social y cultural norte/sur declaraban que: "We realize that the liberation of all oppressed peoples necessitates the destruction of the political-economic systems of capitalism and imperialism as well as patriarchy ${ }^{8 \prime}$.

Las editoras de la primera antología de mujeres de color "This Bridge We Called my back" han reconocido el manifiesto feminista del Combahee River Collective como el punto de partida de la ideología post-feminista donde los pilares post-coloniales de la conciencia crítica dictaminan que la fisura por la que se desangra la utopía de unidad feminista, no puede ser apaleada por la misma mentalidad que la ha creado.

En su introducción de 1981, tanto Gloria Anzaldúa como Cherrie Moraga dilucidaban los límites inclusivos de una nueva categoría de concienciación política:

"We want to express to all women -especially to white middle-class women- the experiences which divide us as feminists; we want to examine incidents of intolerance, prejudice and denial of differences within the feminist movement. We intend to explore the causes and sources of, and solutions to these divisions. We want to create a definition that expands what "feminism" means to us" (III).

Enfatizando en el hecho de que sin la autonomía política de grupos oprimidos, cualquier política de coalición ${ }^{9}$ o movimiento de liberación está abocada al fracaso, prometían que el feminismo del "tercer mundo" se encargaría de alimentar a la gente en todas sus hambres ("is about feeding people in all their hungers"10).

Pero en la exploración de la democratización de las diferencias culturales, el rechazo de ciertas mujeres a identificarse como "feministas" supone el punto de fricción más grande a la que se enfrenta el feminismo dentro de sus propias filas.

\section{Moraga y Anzaldúa 1981: 234}

8 Ibid. 237.

9 Moraga, Cherríe Loving in the War years Loving in the War Years: Lo que nunca pasó por sus labios. Cambridge, MA South End Press, 2000:124.

10 Ibid.p.123.
Fue también en la década de los 80s, cuando Li Xiaojiang, pionera en el campo de los "Estudios de Mujeres" concibió ella misma esta disciplina en la ciudad de Zhengzhou, provincia de Henan, China. Basándose en la pretensión reduccionista del feminismo anglo-europeo que reduce la opresión a una cuestión de género, Li Xiaojiang, al igual que otras voces intelectuales chinas, ha afirmado con rotundidad que el feminismo (nüxing Zhuyi or nüquan zhuyi) no existía en China y que ellas ni se identificaba con el feminismo ni se consideran feministas ${ }^{11}$.

A ojos del feminismo anglo-europeo, estas declaraciones levantan barricadas contra la posibilidad de un diálogo intercultural feminista al tiempo que fragmentan el pensamiento tradicional del feminismo como un discurso en contra de la institución del estados-naciones patriarcales. Sin embargo, lo que la postura de Li Xiaojiang reclama verdaderamente es la ignorancia profunda del contexto del socialismo en china y la falta de humildad y curiosidad por parte del "sujeto occidental" de posicionarse en esa realidad histórico-social.

Cuando el estado socialista subió al poder en 1949, la Ley del Matrimonio (1950) legalizó institucionalmente la igualdad entre hombres y mujeres que quedó fraguada en la Constitución China de 1954 garantizando la igualdad de derechos de las mujeres en todas las esferas sociales y políticas ${ }^{12}$. Es entonces cuando se creó la “Federación de las Mujeres" una institución intermediaria entre el estado socialista y las mujeres con una supuesta jurisprudencia a nivel rural encargada de velar por los derechos económicos, políticos, culturales y educacionales de las mujeres. En ese sentido, estableciendo una comparación con la situación en occidente, las intelectuales chinas argumentan que muchos de los derechos por los cuales se sigue luchando en países occidentales, se consiguieron en China en los años 50s, tales como "trabajo igual/salario igual", así que el feminismo como discurso que contraataca al estado patriarcal, no era necesario en la china de Mao y post-Mao; de ahí de la irrelevancia del feminismo occidental en ese contexto socialista.

Para entender esta valoración es necesario asumir que, sin el conocimiento del socialismo en China, la posibilidad de la traslación y el entendimiento cultura desemboca en "encounter of incommensurability", entendiéndose lo inconmensurable no como la consecuencia de una postura existencialista o absoluta de la diferencia sino fruto de la ignorancia ${ }^{13}$.

$11 \mathrm{Li}$, Xiaojing .Challenge and Response: Lectures on Women's Studies in the New Period [tiaozhan yu huiying: xin shiqi funü yanjiu jiangxuelu) Zhengzhou: Henan. 1996.

12 Shu-Mei Shih " Ethics of Transcultural Encounters, or "When" Does a "Chinese" Woman Become a "Feminist" en Dialogue and Difference Waller, Marguerite y Sylvia Marcos. Dialogue and Difference: Feminisms Challenge Globalization. New York: Palgrave Macmillan, 2005:7. También ve Yang, 1999: 37

13 Shu-Mei Shih "Ethics of Transcultural Encounters"en Dialogue and Difference. 2005:7 
Escuchando las discrepancias de las intelectuales chinas con respecto al énfasis de occidente en insistir que la dominación masculina es la principal causa de opresión de las mujeres, Christina H. Sommers se pregunta “¿Quién ha robado el feminismo?" en la reflexión de cómo las mujeres en un sentido intercultural, se han traicionado entre ellas mismas. Sommers sugiere solo un "feminismo de igualdad" y no "feminismo de género" podrá unificar la voz intercultural feminista contra opresión social de las mujeres $^{14}$

Es importante reflexionar hasta qué punto el sujeto occidental ha tenido el poder de arbitrar "diferencia y símil" en términos reduccionistas. No hay que olvidar que la crítica al "Orientalismo" demuestra una dinámica de poder implícita en la que se encasilla lo no-occidental como "an alibi for the lack of interest in comprehending the Other in its own terms, reducing the Other to the site of difference to explain away the need to attend to its opacity and complexity"15. Shu-Mei Shih en "la ética en el encuentro intercultural" declara que:

"It is not that the Western feminist has a mistaken notion of difference and with Westem fe is the focut of s the power arbitrarily conferring difference and similarity on the non-western woman"16.

Esto ha desembocado en la crítica de un "cosmopolitismo asimétrico" entre lo occidental-no occidental, que demuestra el imperialismo ideológico por el cual los intelectuales no-occidentales tienen que tener conocimientos de culturas y lenguas de las "metrópolis" para ser considerados "cosmopolitas" mientras que el sujeto occidental no necesita ser sabedor de otras culturas o lenguas para serlo ${ }^{17}$. La asimetría cultural del cosmopolitismo defiere del poder de asumir ignorancia sobre otras culturas y seguir siendo parte de "sujeto dominante".

La negación feminista de las "propias feministas" desemboca en una desgarrada celebración multicultural atrapada en el abismo de la amnesia e ignorancia histórica institucionalizada que separa culturas, tradiciones y lenguajes provocando una falta de comunicación y entendimiento de cómo la revolución feminista ha evolucionado de manera específica dependiendo del contexto cultural desde el cual nos posicionemos.

14 Sommers, Christina Hoff. 1994. Who Stole Feminism?.How Women Have Betrayed Women. New York: Simon \& Schuster. p.22-23.

15 Shu-Mei Shih “ Ethics of Transcultural Encounters" en Dialogue and Difference 2005:5

16 Shu-Mei Shih “ Ethics of Transcultural Encounters" en Dialogue and Difference 2005:5

17 Reflexión sobre la arrogancia cultural y lingüística de países anglo-sajones o europeos no hablan más que el inglés como "lengua internacional". Terminología de Shu-Mei Shih en" Ethics of Transcultural Encounters" en Dialogue and Difference. 2005:4
Lo relevante de estas reflexiones es entender que una verdadera democratización de la multiculturalidad feminista no podrá nunca ser un acercamiento no jerárquico sin un proceso previo de "inter-posicionalidad", entendido como la capacidad de posicionarnos en el lugar socio-político y económico del "Otr@”.

La negación de una identidad "feminista" por parte de la fundadora de "estudios de mujeres" pone de manifiesto como la pretensión universalista del conocimiento "occidental" encasilla las diferencias culturales dentro del concepto restrictivo de "lo otr@". La ignorancia intercultural de los factores histórico-políticos que definen las diferentes versiones del feminismo es lo que hace que intelectuales como Li se nieguen a ser "categorizadas étnicamente" por el alcance global del feminismo de occidente y que denuncien que el único modo en el que feminismo occidental contiene las diferencias culturales es a través del "multiculturalismo".

El multiculturalismo, desde las lentes del feminismo "del tercer mundo", divide y trata a los intelectuales no-occidentales en relación a dos parámetros:

a)"Etnicidad recalcitrante", como la representación de la diferencia absoluta o “Otr@”;

b)"minoría étnica asimilada", como sería por ejemplo el caso de las "feministas de la diáspora"18.

Demostrar que no podrá haber nunca un entendimiento transcultural sin antes sanar la ignorancia que tenemos de nuestros contextos es dar un paso hacia delante en la conciencia crítica de la multiculturalidad feminista.

Es entonces cuando dentro de este proceso de democratización intercultural, los nuevos horizontes del pensamiento fronterizo de la conciencia mestiza de Gloría Alzandúa, en Borderlands/La Frontera: The New Mestiza ${ }^{19}$ irrumpieron en la catarsis descolonizadora de la ideología feminista, recordándonos que no existen más fronteras que las que nosostr@s mism@s nos queramos levantar.

Dando voz a nuevas imágenes de identidad, parafraseando a Virginia Wolf y al pragmatismo universalista del feminismo occidental en "La encrucijada / The Crossroads" Gloria Alzandúa reafirma:

"As a mestiza...I have no country.....I am cultureless because, as a feminist, I and Anglos collective cultural /religious I ale- derich another culture, a new story to explain the world and our participation in it, a new

18 En Shu-Mei Shih “ Ethics of Transcultural Encounters” en Dialogue and Difference 2005: 19 19 Anzaldúa, Gloria E. Borderlands/La Frontera: The New Mestiza. San Francisco: Aunt Lute, 1987. 
value system with images and symbols that connect us to each other and to the planet ${ }^{20 \prime}$

La fuerza del pensamiento fronterizo emana del poder de erigir nuevas teorías que rescriban la historia de un feminismo sin fronteras.

Tras décadas de un feminismo del "tercer mundo" defendido como aparato crítico, teoría y método ${ }^{21}$ se podría afirmar que su aliento de transformación ha supuesto un antes y un después en la apertura de un diálogo intercultural no jerárquico.

Su legado es en sí una nueva conciencia crítica que anhela construir puentes postfeministas a partir de un proceso de democratización e "interposicionalidad" de las diferencias culturales. A través de las diferentes ediciones de This Bridge Called My Back: Writings by Radical Women of Color, el debate post-colonial se alzó como el clamor de la conciencia histórica que dice "basta ya": dejemos de mimetizar y disfrazar de académico dinámicas de poder implícitas en el acercamiento de las diversas voces que configuran el conglomerado multicultural feminista.

Sin embargo, después de todo y después de tanto, el movimiento feminista, como "movimiento intercultural unificado", sigue sin haber existido todavía.

Es necesario dar un paso hacia delante en la conciencia crítica y entender que la unificación no es sinónimo de asimilación, sino de coalición.

Pero ese paso no podrá darse sin antes calibrar como la gran ironía dentro de las aportaciones del feminismo del "tercer mundo" es que, si realmente aplicamos la democratización e "interposicionalidad" como la logística de una identidad feminista intercultural, lo inclusivo se tiñe de tintes exclusivistas. La misma conciencia crítica que las mujeres de color revindican en el análisis de las dinámicas de poder implícitas dentro del diálogo intercultural norte/sur, demuestra que la categoría política de "mujeres de color" es por definición "sexista y excluyente" con respecto a los hombres y aquell@s ciudadan@s de "no-color"; del mismo modo, 1a significación "tercer mundo" es también excluyente de un "primer y segundo mundo", así como una corroboración neoimperialista de que "sí, existe un tercer mundo". Incluso la conciencia mestiz@ establece una división subjetiva con aquellos grupos sociales que no se identifiquen con la significación implícita de lo "mestizo", además que desde la perspectiva de "género" cabría rebautizar lo mestizo como "mestiz@", abrazando la democratización tanto de lo femenino como de lo masculino, en el proyecto común de una nueva multiculturalidad.

20 Ibid. p.102-3.

21 Chela Sandoval, "Mestizaje as Method: Feminst-of-Color Challenge the Canon" en Carla Trujillo, eds. Living Chicana Theory. Berkeley, CA: Third Woman Press, 1998:353.
No se puede luchar contra categorías políticamente dominantes, levantando otras. Y así como el conseguir anular todas las dinámicas de poder en el diálogo feminista, solo podrá ser posible a través de un proceso de democratización e "interposicionalidad", la radicalización y polarización del pensamiento feminista solo puede dejar a un lado sus contradicciones ideológicas redefiniéndose en el más inclusivo de todos sus enfoques: la conciencia planetaria de un feminismo sin fronteras.

La conciencia planetaria se basa en la especificidad cultural como la riqueza de la naturaleza humana. Lo planetario, como parte de una terminología feminista descolonizadora e inclusiva, está, por definición, incompleto sin la unión (que no es fusión) de todas las manifestaciones culturales que conforman este planeta, desarrollando así nuevos caminos hacia la disipación de los rencores que mantienen abiertas nuestras cicatrices feministas.

De las palabras de Audre Lorde 22 :"Difference is that raw material and powerful connection from which our personal power is forged" emana el poder de transformar tantas divisiones feministas en una unidad no-jerárquica, en donde la conciencia planetaria es lo más cerca que podremos llegar de alcanzar entre tod@s un feminismo sin fronteras.

\section{REFERENCIAS BIBLIOGRÁFICAS}

Anzaldúa, G. E., Borderlands/La Frontera: The New Mestiza, San Francisco: Aunt Lute, 1987.

George, Rosemary M. "Holmes in the Empire, Holmes in the Home", en Burning Down the House. Recycling Domesticity, Columbia Press, 1998

Li, X., Challenge and Response: Lectures on Women's Studies in the New Period [tiaozhan yu huiying: xin shiq funü yanjiu jiangxuelu], Zhengzhou, Henan, 1996.

Lorde, A., Sister Outsider. Essays and Speeche, Trumansberg, NY, Crossing Press, 1984.

Moraga, C., and G. Anzaldúa (eds.), This Bridge Called My Back: Writings by Radical Women of Color, 1981, New York, Kitchen Table/Women of Color Press, 1983

----, Loving in the War Years: Lo que nunca pasó por sus labios, Cambridge, MA, South End Press, 2000.

Sandoval, C., "Mestizaje as Method: Feminst-of-Color Challenge the Canon", en Carla Trujillo, eds. Living Chicana Theory, Berkeley, CA: Third Woman Press, 1998, 353.

Shih, Shu-Mei "Ethics of Transcultural Encounters, or "When" Does a "Chinese" Woman Become a Feminist", en Dialogue and Difference, 2005, 4-28.

Sommers, C. H., Who Stole Feminism? How Women Have Betrayed Women, New York, Simon \& Schuster,

22 Audre, Lorde,,Sister Outsider.Essays and Speeches. Trumansberg, NY: Crossing Press, 1984:112 
Waller, M. y Sylvia, M., Dialogue and Difference: Feminisms Challenge Globalization, New York, Palgrave Macmillan, 2005. 